\title{
Intensivtherapie bei HIV-Infektion
}

B. Salzberger

66.1 Einleitung -836

66.2 Diagnostik und Stadien der HIV-Infektion - 836

66.2.1 Diagnostik - 836

66.2.2 Stadieneinteilung -836

66.3 Antiretrovirale Therapie auf der Intensivstation - 836

66.3.1 Einleitung und Fortsetzung der antiretroviralen Therapie - 836

66.3.2 Inflammatorisches Immunrekonstitutionsyndrom (IRIS) - 838

66.3.3 Postexpositionsprophylaxe der HIV-Infektion - 838

66.4 Pulmonale Manifestationen der HIV-Infektion - 838

66.4.1 Pneumocystis-jirovecii-Pneumonie - 838

66.4.2 Bakterielle Pneumonien und andere pulmonale Manifestationen - 839

66.5 ZNS-Manifestationen bei HIV-Infektion - 840

66.5.1 Zerebrale Toxoplasmose -840

66.5.2 Kryptokokkenmeningitis - 840

66.5.3 Andere -840

66.6 Gastrointestinale Komplikationen - 840

66.7 Andere Komplikationen - 841

Literatur - 841 


\subsection{Einleitung}

In den ersten Jahren der AIDS-Epidemie war die Behandlung von HIV-assoziierten Komplikationen mit einer ICU-Mortalität von 80-90\% verknüpft [6]. Mit der rascheren Diagnose und besseren Therapie zuerst der opportunistischen Erkrankungen und der HIVInfektion verbesserte sich die Prognose deutlich [9]. Heute ist die Langzeitprognose HIV-infizierter Patienten mit einer wirksamen antiretroviralen Therapie am ehesten vergleichbar mit der anderer chronischer Erkrankungen [1].

Damit hat sich auch das Spektrum der zum Intensivaufenthalt führenden Erkrankungen geändert: Opportunistische Erkrankungen sind seltener und nicht-HIV-assoziierte, wie z. B. kardiovaskuläre Erkrankungen, häufiger geworden. Da sich die intensivmedizinische Betreuung von HIV-infizierten Patienten bei diesen Erkrankungen nicht von denen anderer Patienten unterscheidet, werden hier nur die spezifisch HIV-assoziierten Erkrankungen behandelt.

Opportunistische Erkrankungen sind durch die moderne antiretrovirale Therapie sehr viel seltener geworden, sie treten jedoch immer noch als Primärmanifestation der HIV-Infektion auf und führen dann auch häufig zu einem schweren und intensivpflichtigen Verlauf [12]. Patienten, deren HIV-Infektion erst durch schwere Komplikationen oder bei weit fortgeschrittenem Immundefekt entdeckt wird, machen einen Anteil von ca. 25\% aller Erstdiagnosen der HIV-Infektion aus.

\subsection{Diagnostik und Stadien der HIV-Infektion}

\subsubsection{Diagnostik}

Die Diagnose der HIV-Infektion kann durch Nachweis von Antikörpern oder Virusbestandteilen erfolgen. Sie wird in der Regel gestellt mittels eines ELISA-Tests, der HIV-1- und HIV-2-Antikörper sowie HIV-Antigen nachweisen kann. Die sehr hohe Sensitivität des ELISA bedingt eine niedrige Spezifität, deshalb ist eine Bestätigung im Immunfluoreszenz- bzw. Western-Blot-Test notwendig. Ein direkter Virusnachweis mittels PCR, besonders bei unklarer Serologie und Verdacht auf Primärinfektion, kann ebenfalls die Diagnose sichern.

C Cave

Patienten mit einer HIV-Infektion haben v. a. während der oft langjährigen klinischen Latenzzeit ein völlig unauffälliges Routinelabor. Ein normales Labor schließt eine HIV-Infektion nicht aus! Mögliche Hinweise (meist spät) sind eine Lymphopenie und eine polyklonale Gammopathie.

\subsubsection{Stadieneinteilung}

Die Stadieneinteilung erfolgt nach der Klassifikation der CDC (-Tab. 66.1). Hierzu gehört die Messung der CD4-Zellzahl im peripheren Blut als wichtigster Marker des Immunstatus. Im natürlichen Verlauf der HIV-Infektion schließt sich an eine symptomatische ( $\triangleright$ unten.) oder asymptomatische primäre HIV-Infektion häufig eine langjährige klinische Latenzphase an. Erst bei deutlicher Verminderung der CD4-Zellzahl kommt es dann zum Auftreten von opportunistischen Erkrankungen.

\section{Primäre HIV-Infektion}

Eine symptomatische primäre HIV-Infektion tritt etwa bei $20-30 \%$ aller Infizierten auf. Dabei kommt es 3-6 Wochen nach Erstinfektion $\mathrm{zu}$ einem mononukleoseähnlichen Krankheitsbild mit sehr unterschiedlicher Ausprägung. Neben Fieber, einem generalisierten makulopapulösem Exanthem und schwerem Krankheitsgefühl können auch generalisierte Lymphknotenschwellungen vorhanden sein. Im Labor findet sich eine Lymphozytose mit Reizformen, eine mäßige Erhöhung der Transaminasen und LDH sowie eine Thrombopenie.

Zur Intensivaufnahme können v. a. die neurologischen Komplikationen (Meningitis oder Guillain-Barré-Syndrom), seltener eine Blutungsneigung bei Thrombopenie, führen [11]. Bei schweren Komplikationen durch eine primäre HIV-Infektion kann eine antiretrovirale Therapie erwogen werden, eine Verbesserung der Langzeitprognose durch einen derart frühen Therapiebeginn ist bisher jedoch nicht nachgewiesen [4].

\subsection{Antiretrovirale Therapie auf der Intensivstation}

\subsubsection{Einleitung und Fortsetzung der antiretroviralen Therapie}

Eine Therapie der HIV-Infektion ist mit dem Auftreten einer opportunistischen Erkrankung klar indiziert, ebenso beim Auftreten HIV-assoziierter Symptome. Eine weitere Indikation ist bei asymptomatischen Patienten beim Unterschreiten der Grenze von $350 \mathrm{CD} 4 / \mathrm{mcl}$ gegeben [4]. So ist praktisch immer bei einer HIVassoziierten Erkrankung als Ursache des Intensivaufenthalts die Notwendigkeit einer antiretroviralen Therapie gegeben. Diese muss nicht notfallmäßig sofort, sollte aber rasch, d. h. innerhalb von ca. 10 Tagen nach Auftreten der Komplikation begonnen werden.

Die einzige Ausnahme ist hier die Tuberkulose. Gerade bei der Tuberkulose ist das Risiko einer paradoxen Reaktion (IRIS, $>$ Abschn. 66.3.2) besonders hoch. Deshalb sollte hier in aller Regel die antiretrovirale Therapie frühestens nach ca. 8 Wochen Therapie der Tuberkulose eingesetzt werden. Für die Initialtherapie sind mehrere Kombinationen sinnvoll und empfohlen (•Tab. 66.2).

Eine einmal eingeleitete antiretrovirale Therapie wird auch auf der Intensivstation fortgeführt. Eine Unterbrechung birgt das Risiko für eine Resistenzentwicklung bzw. ein Therapieversagen und sollte nur in gut begründeten Ausnahmesituationen (z. B. schwere Nebenwirkungen) erfolgen. Auf die entsprechenden Neben- und Wechselwirkungen mit anderen Medikamenten muss dabei geachtet werden $[7,10]$ (•Tab. 66.3).

- Tab. 66.1 Staging der HIV-Infektion nach dem Schema der Centers of Disease Control (CDC), USA

\begin{tabular}{|l|l|l|l}
$\begin{array}{l}\text { Klinische Ma- } \\
\text { nifestationen/ } \\
\text { CD4-Zellzahl }\end{array}$ & $\begin{array}{l}\text { A=Asympto- } \\
\text { matisch oder } \\
\text { Lymph- } \\
\text { adenopathie } \\
\text { oder primäre } \\
\text { HIV-Infektion }\end{array}$ & $\begin{array}{l}\text { B=Mindere } \\
\text { klinische } \\
\text { Manifesta- } \\
\text { tion, z. B. } \\
\text { oraler Soor }\end{array}$ & $\begin{array}{l}\text { C=Definitive } \\
\text { opportunisti- } \\
\text { sche Erkran- } \\
\text { kung, z. B. PcP } \\
\text { oder Kaposi- } \\
\text { Sarkom }\end{array}$ \\
\hline $\begin{array}{l}1 \geq 500 \mathrm{CD} 4 / \mathrm{mcl} \\
\mathbf{2}=200-500 \mathrm{CD} 4 / \\
\mathrm{mcl}\end{array}$ & A2 & B1 & C1 \\
\hline $3 \leq 200 \mathrm{CD} 4 / \mathrm{mcl}$ & A3 & B2 & C2 \\
\hline
\end{tabular}


- Tab. 66.2 Antiretrovirale Therapie: Indikationen, Kombinationen und Postexpositionsprophylaxe

Indikationen zur Therapie [4]

Primär empfehlenswerte Kombinationen [4]

Postexpositionsprophylaxe [5]
Klare Indikation:

- Alle Patienten mit symptomatischer HIV-Infektion, z. B. alle mit klinischem Stadium C, nahezu alle mit klinischem Stadium B

- Asymptomatische Patienten mit eingeschränktem Immunsystem, definiert durch CD4-Zellzahl $\leq 350$ CD4/ $\mathrm{mcl}$

Indikation nicht sicher:

- Primäre HIV-Infektion

- Asymptomatische Patienten mit CD4-Zellzahl > 350 CD4/mcl

Kombination zweier Nukleosidanaloga, z. B. Fixkombinationen

- Tenofovir + Emtricitabin oder

- Abacavir ${ }^{\mathrm{a}}+$ Lamivudin

plus

- Nichtnukleosidaler Inhibitor der RT, z. B. Efavirenz

oder

- mit Ritonavir pharmakologisch geboosteter Proteaseinhibitor, z. B. Lopinavir, Fosamprenavir, Darunavir, Atazanavir

Indikation:

- Parenteraler oder Schleimhautkontakt mit potenziell HIV-infiziertem Material, z. B. Nadelstichverletzung, Blutspritzer ins Auge oder auch ungeschützter sexueller Kontakt mit sicher HIV-infizierter Person

Durchführung:

- Tenofovir + Emtricitabin oder

- Zidovudin + Lamivudine

Jeweils plus

- Lopinavir/r (Kombinationspräparat) oder

- Efavirenz

a Vorherige Bestimmung von HLA-B5701 zur Vermeidung des Hypersensitivitätssyndroms.

- Tab. 66.3 In der Intensivmedizin relevante Neben- und Wechselwirkungen einer antiretroviralen Therapie der wichtigsten Substanzen

\begin{tabular}{|c|c|c|c|}
\hline Substanz/-Gruppe & Relevante NW & Metabolismus & $\begin{array}{l}\text { Potenzielle Interaktionen } \\
\text { (Beispiele) }\end{array}$ \\
\hline \multicolumn{4}{|c|}{ Nukleosid/Nukleotidanaloga } \\
\hline Tenofovir & CK $\uparrow$, Kreatinin $\uparrow$ (selten) & Renale Elimination & $\begin{array}{l}\text { Potenziell: } \\
\text { Cotrimoxazol, Aciclovir, Cidofo- } \\
\text { vir Ganciclovir, Amphotericin B } \\
\text { über renale Elimination }\end{array}$ \\
\hline Abacavir & $\begin{array}{l}\text { Hypersensitivitätssyndrom (HLA- } \\
\text { B5701 assoziiert) }\end{array}$ & $\begin{array}{l}\text { Hepatische Elimination (keine } \\
\text { Induktion oder Hemmung } \\
\text { von CYP-Enzymen) }\end{array}$ & $\begin{array}{l}\text { Potenziell: } \\
\text { Rifampicin, Ganciclovir }\end{array}$ \\
\hline Zidovudin & Anämie, Leukopenie & Renale Elimination & $\begin{array}{l}\text { Mögliche Verstärkung von Ne- } \\
\text { benwirkungen mit Ganciclovir, } \\
\text { Zytostatika }\end{array}$ \\
\hline Emtricitabin & NW sehr selten, Schlafstörungen & Renale Elimination & Kaum Interaktionen \\
\hline Lamivudin & NW sehr selten, Schlafstörungen & Renale Elimination & Kaum Interaktionen \\
\hline \multicolumn{4}{|l|}{ NNRTI } \\
\hline Efavirenz & $\begin{array}{l}\text { Schlafstörungen, Hautausschlag, } \\
\text { Depression (selten), Leberenzyme } \uparrow\end{array}$ & $\begin{array}{l}\text { Hepatisch metabolisiert } \\
\text { durch CYP } 3 \text { A4 u. a. (Indukti- } \\
\text { on und Inhibition) }\end{array}$ & $\begin{array}{l}\text { Rifampicin, Statine, Coumarine } \\
\text { u. a. }\end{array}$ \\
\hline Nevirapin & Hautausschlag, Leberenzyme $\uparrow$ & $\begin{array}{l}\text { Hepatisch metabolisiert } \\
\text { durch CYP } 3 \text { A4 u. a. (Indukti- } \\
\text { on und Inhibition) }\end{array}$ & $\begin{array}{l}\text { Rifampicin, Statine, Coumarine } \\
\text { u. a. }\end{array}$ \\
\hline \multicolumn{4}{|c|}{ Proteaseinhibitoren ${ }^{a}$} \\
\hline Lopinavir/r & $\begin{array}{l}\text { Diarrhö } \\
\text { Triglyzeride } \uparrow \\
\text { Cholesterin } \uparrow \\
\text { Leberenzyme } \uparrow\end{array}$ & $\begin{array}{l}\text { Hepatisch metabolisiert, } \\
\text { v. a. durch CYP 3A4, starke } \\
\text { Inhibition }\end{array}$ & $\begin{array}{l}\text { Rifampicin, Statine, Coumari- } \\
\text { ne, Azole, Ciclosporin u. a. }\end{array}$ \\
\hline
\end{tabular}


- Tab. 66.3 Fortsetzung

\begin{tabular}{|c|c|c|c|}
\hline Substanz/-Gruppe & Relevante NW & Metabolismus & $\begin{array}{l}\text { Potenzielle Interaktionen } \\
\text { (Beispiele) }\end{array}$ \\
\hline Fosamprenavir/r & $\begin{array}{l}\text { Diarrhö } \\
\text { Triglyzeride } \uparrow \\
\text { Cholesterin } \uparrow \\
\text { Leberenzyme } \uparrow\end{array}$ & $\begin{array}{l}\text { Hepatisch metabolisiert, } \\
\text { v. a. durch CYP 3A4, starke } \\
\text { Inhibition }\end{array}$ & $\begin{array}{l}\text { Rifampicin, Statine, Coumari- } \\
\text { ne, Azole, Ciclosporin u. a. }\end{array}$ \\
\hline Darunavir/r & $\begin{array}{l}\text { Diarrhö (seltener) } \\
\text { Triglyzeride } \uparrow \\
\text { Cholesterin } \uparrow \\
\text { Leberenzyme } \uparrow\end{array}$ & $\begin{array}{l}\text { Hepatisch metabolisiert, } \\
\text { v. a. durch CYP 3A4, starke } \\
\text { Inhibition }\end{array}$ & $\begin{array}{l}\text { Rifampicin, Statine, Coumari- } \\
\text { ne, Azole, Ciclosporin u. a.,. }\end{array}$ \\
\hline Atazanavir/r & $\begin{array}{l}\text { Diarrhö (seltener) } \\
\text { Triglyzeride } \uparrow \\
\text { Cholesterin } \uparrow \text { (seltener) } \\
\text { Leberenzyme } \uparrow\end{array}$ & $\begin{array}{l}\text { Hepatisch metabolisiert, } \\
\text { v. a. durch CYP 3A4, starke } \\
\text { Inhibition }\end{array}$ & $\begin{array}{l}\text { Rifampicin, Statine, Couma- } \\
\text { rine, Azole, Ciclosporin u. a., } \\
\text { Inhibition von Adsorption bei } \\
\text { gleichzeitiger PPI-Gabe }\end{array}$ \\
\hline
\end{tabular}

a Jeweils mit pharmakologischer Boosterung.

Für potenzielle Interaktionen Interaktionsdatenbanken konsultieren [7].

\subsubsection{Inflammatorisches Immunrekonstitutionsyndrom (IRIS)}

Kurz nach Einführung der antiretroviralen Kombinationstherapie wurden bei einigen Patienten unübliche klinische Verläufe von opportunistischen Erkrankungen unter einer eingeleiteten antiretroviralen Therapie beobachtet. Mittlerweile sind solche Verläufe für nahezu alle opportunistischen und sogar für Autoimmunerkrankungen beschrieben. Allen diesen Verläufen ist eine paradoxe klinische Verschlechterung nach Beginn einer antiretroviralen Therapie gemein, z. B. einer Verschlechterung einer Tuberkulose oder auch das Auftreten einer neuen opportunistischen Infektion bzw. einer Autoimmunerkrankung. Alle diese Verläufe werden unter dem Begriff des inflammatorischen Immunrekonstitutionssyndroms (IRIS) zusammengefasst.

Das Risiko eines IRIS ist besonders hoch, wenn der initiale Immundefekt schwer war und der Zeitpunkt des Auftretens mit einem raschen Anstieg der CD4-Zellzahl im Blut korreliert. Nach dem Verlauf und den gemessenen Zytokinmustern muss am ehesten von einer Aktivierung des angeborenen Immunsystems ausgegangen werden. Welcher genaue Pathomechanismus diesem Syndrom zugrunde liegt, ist aber bisher ungeklärt. Am häufigsten ist eine solche paradoxe klinische Verschlechterung beim Vorliegen einer Tuberkulose, sie kann jedoch auch die Therapie einer PcP komplizieren [2].

Eine prophylaktische Therapie mit Glukokortikoiden reduziert das Risiko und den Schweregrad des IRIS bei gleichzeitiger Tuberkulose, kann aber nicht generell empfohlen werden.

( Cave

Bei einer Verschlechterung einer opportunistischen Infektion im zeitlichen Zusammenhang mit der Einleitung einer antiretroviralen Therapie muss neben einem Therapieversagen auch das Auftreten eines IRIS in Betracht gezogen werden.

\subsubsection{Postexpositionsprophylaxe der HIV-Infektion}

Routinemaßnahmen zur Vermeidung von Infektionen durch Blutbestandteile sind Bestandteil der Hygienemaßnahmen auf jeder Intensivstation. Diese Maßnahmen verhindern auch die Übertragung des HIV mit hoher Sicherheit. Unfälle mit einem parenteralen oder Schleimhautkontakt mit HIV-kontaminiertem Material stellen ein Risiko für eine HIV-Übertragung dar (Übertragung in ca. 0,03\% von Fällen mit parenteralem Kontakt). Die Art der Verletzung, das kontaminierte Material und vermutlich auch die Höhe der VirusRNA im Blut haben dabei Einfluss auf das Risiko. So ist z. B. eine perkutane Verletzung mit einem höheren Risiko als ein Schleimhautkontakt verbunden, eine Verletzung mit einer Hohlnadel gefährlicher als eine mit einer Nahtnadel.

Aus Kohortenstudien ist bekannt, dass eine Postexpositionsprophylaxe mit antiretroviralen Substanzen das Risiko einer Infektion um ca. $80 \%$ mindert, deshalb sollte nach lokalen Maßnahmen eine solche, bestehend aus einer antiretroviralen Dreifachkombination nach entsprechendem Kontakt, angeboten und für 4 Wochen appliziert werden [5] (•Tab. 66.2).

\section{Tipp}

Bei beruflicher HIV-Exposition sollte dringend ein BG-Verfahren eingeleitet werden, damit eine Dokumentation des Unfalls erfolgt und entsprechende Nachuntersuchungen durchgeführt werden. Dies dient v. a. der Sicherheit der Beschäftigten.

\subsection{Pulmonale Manifestationen der HIV-Infektion}

\subsubsection{Pneumocystis-jirovecii-Pneumonie}

Die häufigste pulmonale Manifestation der HIV-Infektion ist die Pneumocystis-jirovecii-Pneumonie (PcP). Sie ist außerdem der häufigste Grund für eine Intensivaufnahme bei HIV-Infizierten. Sie tritt auf bei fortgeschrittenem Immundefekt $\left(<200 \mathrm{CD}_{4} / \mathrm{mcl}\right)$.

Die Symptome sind Fieber, Dyspnoe und trockener Husten. Im Labor findet sich häufig eine Erhöhung der LDH, grob korreliert 

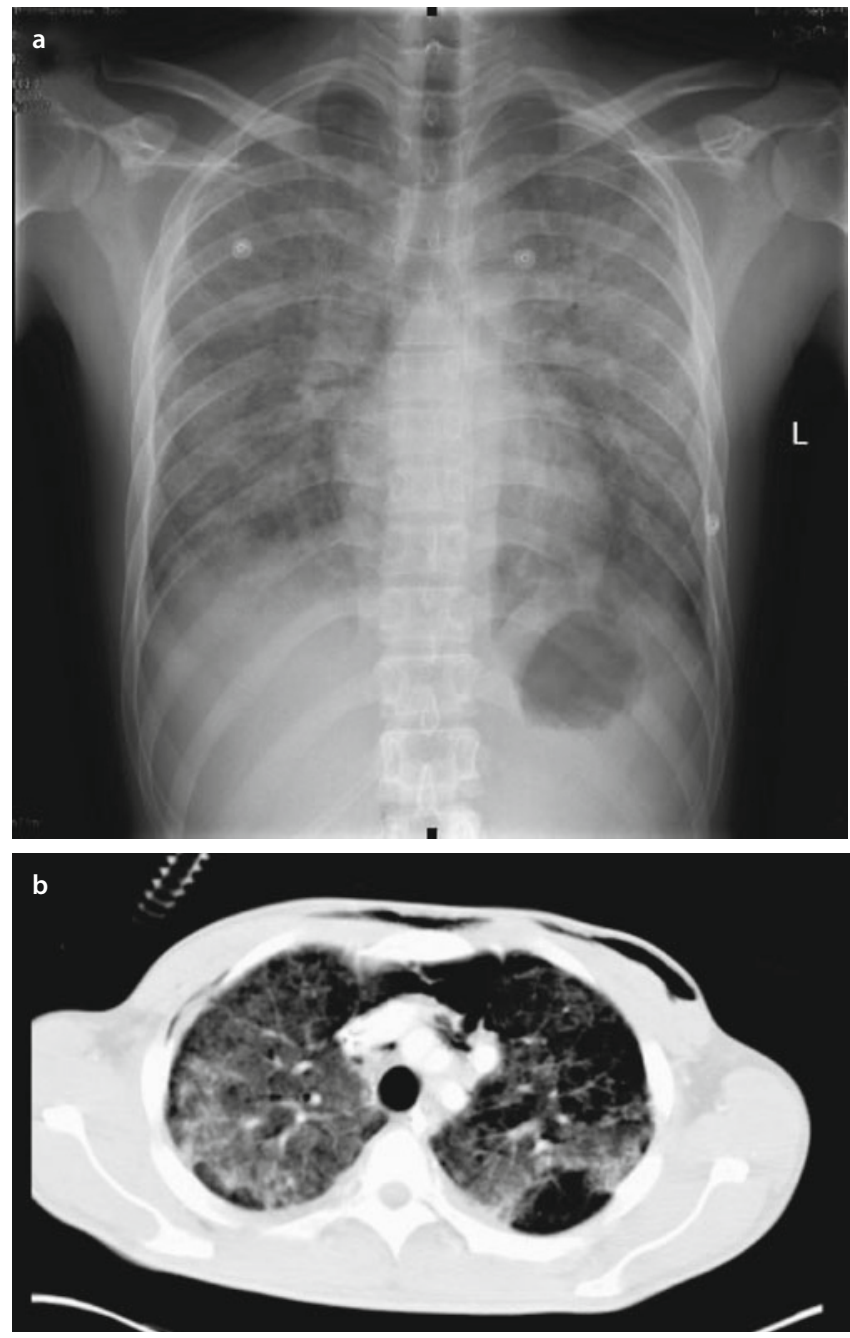

- Abb. 66.1 Thoraxröntgenaufnahme (a) bzw. Computertomographie des Thorax (b) bei einem Patienten mit HIV-Infektion und Pneumocystisjirovecii-Pneumonie (Abb. von Prof. Dr. S. Feuerbach, Institut für Radiologie, Universitätsklinikum Regensburg, mit frdl. Genehmigung) mit dem Schweregrad. Radiologisch zeigt sich anfangs eine geringe interstitielle Zeichnungsvermehrung, später ein ausgedehntes, meist bihilär schmetterlingsförmig konfiguriertes Infiltrat. Eine Computertomographie ist dabei sensitiver als die Übersichtsaufnahme (• Abb. 66.1).

Pneumocystis jirovecii kann mittels Zytologie bzw. PCR aus der Bronchiallavage nachgewiesen werden. Trotz Fortschritten in der Diagnostik und Therapie liegt die ICU-Mortalität der beatmungspflichtigen PcP bei etwa 25\%. Prognostisch ungünstig ist dabei ein hoher Anteil von Granulozyten in der BAL oder ein gleichzeitiger Nachweis von CMV-Virus.

Die Standardtherapie ist hochdosiertes Cotrimoxazol, alternativ Pentamidin (-Tab. 66.4). Bei schweren Verläufen verbessert eine adjuvante Therapie mit Glukokortikoiden die Prognose. Ob bei Nachweis von CMV in der BAL eine antivirale Therapie sinnvoll ist, ist nicht klar.Viele Experten sind jedoch der Ansicht, dass dies nicht notwendig und aufgrund der zusätzlichen Toxzität auch nicht sinnvoll ist. Generell sind genuine CMV-Pneumonien bei der HIVInfektion eine extreme Rarität und auch deshalb der pathogenetische Wert eines Nachweises von CMV-Virus oder -DNA in der BAL in dieser Situation unklar.

Als Komplikation sind bei der PcP häufig Pneumatozelen vorhanden, die für einen Pneumothorax prädisponieren. Obwohl hierfür keine speziellen Studien vorhanden sind, sollte eine Beatmung bei PcP nach den gängigen Standards des ARDS (mit niedrigen Tidalvolumina) erfolgen $[3,8]$

\subsubsection{Bakterielle Pneumonien und andere pulmonale Manifestationen}

Die Häufigkeit von bakteriellen Pneumonien ist bei der HIV-Infektion deutlich erhöht. Die häufigsten Erreger sind S. pneumoniae und $\mathrm{H}$. influenzae. Da hier P. aeruginosa und S. aureus häufige Pathogene sind, sollten diese Erreger bei der empirischen Therapie ebenfalls berücksichtigt werden $[3,8]$.

Andere spezifische Ursachen für ein respiratorisches Versagen bei HIV-infizierten Patienten können eine Infektion mit M. tuberculosis ( Kap. 65), Pilzpneumonien, ein Non-Hodgkin-Lymphom,

- Tab. 66.4 Therapie spezifischer Manifestationen der HIV-Infektion

\begin{tabular}{|c|c|c|}
\hline Manifestation & \multicolumn{2}{|l|}{ Therapieschema } \\
\hline \multirow{4}{*}{$\begin{array}{l}\text { Pneumocystis-jiro- } \\
\text { vecii-Pneumonie }\end{array}$} & Therapie der 1. Wahl & Cotrimoxazol (15-20 mg/kg KG Trimethoprim/Tag) \\
\hline & Alternative & Pentamidin 4 mg/kg KG i.v. (je über 21 Tage) \\
\hline & Adjuvante Therapiemaßnahmen & $\begin{array}{l}\text { Adjuvante Steroidtherapie mit } 2 \times 40 \mathrm{mg} \text { Prednison (bei arteriellem } \mathrm{pO}_{2} \leq 70 \mathrm{~mm} \mathrm{Hg} \\
\text { oder AAd } \mathrm{O}_{2}>35 \mathrm{~mm} \mathrm{Hg} \text { ): } \\
=2 \times 40 \mathrm{mgTag} 1-5, \\
-40 \mathrm{mg} \text { Tag } 6-10 \\
-20 \mathrm{mg} \text { Tag } 11-15 \\
\text { Beatmung mit niedrigem Tidalvolumen (wie bei ARDS) }\end{array}$ \\
\hline & Rezidivprophylaxe & $\begin{array}{l}\text { Cotrimoxazol (verschiedene Optionen der Dosierung), bis CD } 4>200 \text { und HIV-RNA } \\
\text { unter der Nachweisgrenze für } 3 \text { Monate }\end{array}$ \\
\hline \multirow{4}{*}{$\begin{array}{l}\text { Zerebrale Toxoplas- } \\
\text { mose }\end{array}$} & Therapie der 1. Wahl & Pyrimethamin 50-100 mg/Tag + Sulfadiazin 4×1-1,5 g/Tag \\
\hline & Alternative & Pyrimethamin 50-100 mg/Tag + Clindamycin 4×600 mg/Tag für 42 Tage \\
\hline & Adjuvante Therapiemaßnahmen & Folinsäure $10-30 \mathrm{mg} / \mathrm{Tag}$ \\
\hline & Rezidivprophylaxe & $\begin{array}{l}\text { Pyrimethamin } 50 \mathrm{mg} / \mathrm{Tag}+ \\
\text { Sulfadiazin } 4 \times 0,5-1 \mathrm{~g}\end{array}$ \\
\hline
\end{tabular}


- Tab. 66.4 Fortsetzung

\begin{tabular}{|c|c|c|}
\hline Manifestation & \multicolumn{2}{|l|}{ Therapieschema } \\
\hline \multirow{4}{*}{$\begin{array}{l}\text { Kryptokokken- } \\
\text { meningitis }\end{array}$} & Therapie der 1. Wahl & Amphotericin B 0,7 mg/kg KG/Tag + Flucytosin $4 \times 25$ mg/kg KG/Tag für 14 Tage \\
\hline & Alternative & $\begin{array}{l}\text { Fluconazol } 400-800 \mathrm{mg}+ \\
\text { Flucytosin } 4 \times 25 \mathrm{mg} / \mathrm{kg} \mathrm{KG} / \mathrm{Tag} \text { für } 14 \text { Tage } \\
\text { Danach jeweils Fluconazol } 400 \mathrm{mg} \text { für } 8 \text { Wochen }\end{array}$ \\
\hline & Adjuvante Therapiemaßnahmen & Entlastung Liquordruck nach Monitoring (bis Öffnungsdruck <200 mm H $\mathrm{m}_{2} \mathrm{O}$ ) \\
\hline & Rezidivprophylaxe & $\begin{array}{l}\text { Fluconazol } 200 \text { mg/Tag p.o., bis CD >200 und HIV-RNA unter der Nachweisgrenze für } \\
3 \text { Monate }\end{array}$ \\
\hline \multirow{4}{*}{$\begin{array}{l}\text { CMV-Enzephalitis/ } \\
\text { Gastroenteritis/ } \\
\text { Kolitis }\end{array}$} & Therapie der 1 . Wahl & $\begin{array}{l}\text { Jeweils für } 21 \text { Tage } \\
\text { - Ganciclovir } 2 \times 5 \mathrm{mg} / \mathrm{kg} \mathrm{KG/Tag} \mathrm{i.v.}\end{array}$ \\
\hline & Alternative & Foscarnet $2 \times 90 \mathrm{mg} / \mathrm{kg} \mathrm{KG} / \mathrm{Tag}$ i.v. \\
\hline & Adjuvante Therapiemaßnahmen & - \\
\hline & Rezidivprophylaxe & $\begin{array}{l}\text { Nicht etabliert, möglich: } \\
\text { Valganciclovir } 480 \text { mg/Tag, bis CD4 >100 und HIV-RNA unter der Nachweisgrenze } \\
\text { für } 3 \text { Monate }\end{array}$ \\
\hline
\end{tabular}

eine HIV-assoziierte pulmonale Hypertonie oder eine kardiale Dekompensation bei HIV-assoziierter Kardiomyopathie darstellen.

\subsection{ZNS-Manifestationen bei HIV-Infektion}

\subsubsection{Zerebrale Toxoplasmose}

Die zerebrale Toxoplasmose entsteht durch eine Reaktivierung intrazerebraler Toxoplasmoseherde. Eine solche Reaktivierung kann vorkommen bei deutlich erniedrigter $\mathrm{CD}_{4}$-Zellzahl (in der Regel $<150 \mathrm{CD} 4 / \mathrm{mcl}$ ).

Die Symptome entstehen durch die Entzündung und Raumforderung. Sie verursachen fokale neurologische Symptome je nach Lokalisation, die meist plötzlich auftreten, seltener Anfälle und systemische Symptome. Die Diagnose wird initial klinisch gestellt durch eine zerebrale Bildgebung (CT bzw. MRT mit Kontrast). Hier zeigen sich ringförmige kontrastmittelanreichernde raumfordernde Läsionen mit perifokalem Ödem. Morphologisch sind diese Läsionen nicht sicher unterscheidbar von einem zerebralen Non-Hodgkin-Lymphom.

Falls die Erkrankung auf eine antiparasitäre Therapie (•Tab. 66.4) nicht anspricht, ist eine stereotaktische Biopsie indiziert [3].

\subsubsection{Kryptokokkenmeningitis}

Die Kryptokokkenmeningitis ist eine schwer verlaufende Meningitis bei weit fortgeschrittenem Immundefekt $\left(\mathrm{CD}_{4}<100 / \mathrm{mcl}\right)$. Symptome sind Fieber und Kopfschmerzen, ein Meningismus kann vorhanden sein. Der Erreger, Cryptococcus neoformans, ist ubiquitär und verursacht zunächst pulmonale, später disseminierte Infektionen. Die Diagnose wird durch Liquorpunktion gestellt, dabei ist neben der Kultur und dem Nachweis des Kryptokokkenantigens auch ein direkter Nachweis durch Tuschefärbung möglich.

Die Therapie der 1. Wahl besteht aus der Kombination von Amphotericin B und Flucytosin, alternativ Fluconazol (•Tab. 66.4). Eine wichtige adjuvante Therapiemaßnahme bei der Kryptokokkenmeningitis ist die Therapie des meist deutlich erhöhten Liquordrucks durch Punktion nach Druckmonitoring [3].

\subsubsection{Andere}

Virale Enzephalitiden können durch das assoziierte Koma oder andere schwere neurologische Störungen zur Aufnahme auf die Intensivstation führen. Hier sind v. a. Enzephalitiden mit JC-Virus, CMV, HSV und VZV zu nennen. Während die Enzephalitiden durch Herpesviren häufiger Anfälle und schwere Bewusstseinsstörungen verursachen, präsentiert sich die JC-Virusenzephalitis (auch progressive multifokale Leukenzephalopathie, $\mathrm{PML}$ ) häufiger mit kognitiven und fokalen neurologischen Störungen. Der Nachweis der Erreger gelingt durch Liquorpunktion und PCR. In der Bildgebung zeigen sich bei den Enzephalitiden durch Herpesviren meist einzelne fokale Läsionen, während die ausgeprägten entzündlichen Veränderungen bei der PML fast pathognomonisch sind.

Die Enzephalitiden mit HSV und VZV werden nach den bekannten Schemata behandelt, für die Therapie der CMV-Enzephalitis ist Ganciclovir die 1. Wahl, Foscarnet und Cidofovir sind Alternativen. Eine Therapie der PML ist nicht durch Studien etabliert, in vitro wirkt Cidofovir auf JC-Virus.

Eine genuine HIV-Enzephalopathie ist v. a. durch schwerste kognitive Einbußen apparent. Im Liquor zeigt sich ein hoher Nachweis von HIV-RNA und in der Bildgebung eine ausgeprägte Erweiterung der äußeren und inneren Liquorräume. Die Therapie der Wahl ist die antiretrovirale Therapie, durch die oft eine fast vollständige Remission der Klinik erzielt werden kann [3].

\subsection{Gastrointestinale Komplikationen}

Gastrointestinale Blutungen und seltener Perforationen können durch CMV-Ulzerationen im Ösophagus, Magen, Kolon und seltener Dünndarm auftreten. Endoskopisch zeigen sich ausgestanzte multiple Ulzerationen. Blutungen können ebenfalls durch Schleimhautbefall von Kaposi-Sarkomen entstehen. Eine Remission mukokutaner Kaposi-Sarkome kann durche eine antiretrovirale Therapie weitestgehend gelingen. Nur bei Progression bzw. Nichtansprechen sollte eine zytostatische Therapie angewandt werden.

Hepatitiskoinfektionen v. a. mit HCV sind bei der HIV-Infektion häufig und mit einer rascheren Progression zum Leberversagen verbunden. Die Behandlung von hepatologischen Komplikationen 
unterscheidet sich jedoch nicht von der anderer Patienten, eingeschlossen die Lebertransplantation als Ultima ratio [3].

\subsection{Andere Komplikationen}

Hämatoonkologische Manifestationen der HIV-Infektion können ebenfalls zum Intensivaufenthalt führen. Das Risiko für Non-Hodgkin-Lymphome ist für nicht antiretroviral behandelte HIV-infizierte Patienten ungefähr um das 20o-Fache erhöht. Spezielle Formen, die bei der HIV-Infektion häufiger sind, beinhalten das primäre Pleura- bzw. peritoneale Lymphom, das mit HHV-8 assoziiert ist. Ebenso HHV-8-assoziiert ist das Kaposi-Sarkom, das neben Hautund Schleimhäuten auch innere Organe (Leber, Milz u. a.) befallen kann.

\section{Literatur}

1 The Antiretroviral Therapy Cohort Collaboration (2008) Life expectancy of individuals on combination antiretroviral therapy in high-income countries: a collaborative analysis of 14 cohort studies Lancet 372: 293-9

2 Behrens GM, Meyer D, Stoll M et al. (2000) Immune reconstitution syndromes in human immuno-deficiency virus infection following effective antiretroviral therapy Immunobiology 202: 186-93

3 Benson CA, Kaplan JE, Masur H et al. (2004) Treating Opportunistic Infections Among HIV Infected Adults and Adolescents. National Center for HIV, STD and Tb Prevention

4 Deutsche AIDS Gesellschaft (DAIG) (2008) Leitlinien zur antiretroviralen Therapie im Erwachsenenalter, 19.01.2010 [erhältlich unter: www.daignet.de/site-ocntent/hiv-therapie/leitlinien-1]

5 Deutsche AIDS Gesellschaft (DAIG) (2008) Postexpositionelle Prophylaxe der HIV-Infektion, 19.01.2010 [erhältlich unter:www.daig-net.de/siteocntent/hiv-therapie/leitlinien-1]

6 Gatell JM, Marrades R, el-Ebiary M et al. (1996) Severe pulmonary infections in AIDS patients Semin Respir Infect 11: 119-28

7 Liverpool HIV Pharmacology Group (2010) HIV Drug Interactions. Liverpool University [www.hiv-druginteractions.org/]

8 Masur H (2006) Management of patients with HIV in the intensive care unit Proc Am Thorac Soc 3: 96-102

9 Morris A, Wachter RM, Luce J et al. (2003) Improved survival with highly active antiretroviral therapy in HIV-infected patients with severe Pneumocystis carinii pneumonia AIDS 17: 73-80

10 Piscitelli SC, Galliciano KD (2001) Interactions among drugs for HIV and Opportunistic Infections N Engl J Med 344: 985-96

11 Tindall B, Hing M, Edwards P et al. (1989) Severe clinical manifestations of primary HIV infection AIDS 3: 747-9

12 Vincent B, Timsit JF, Auburtin M et al. (2004) Characteristics and outcomes of HIV-infected patients in the ICU: impact of the highly active antiretroviral treatment era Intensive Care Med 30: 859-66

\section{Internetadressen}

Frei zugängliches Lehrbuch zu HIV und Aids [www.hiv-net.de]

Deutsche AIDS-Gesellschaft e. V. mit Therapieleitlinien und vielen weiteren Informationen [www.daig-net.de]

University of California, San Francisco. Webbasiertes Lehrbuch zu HIV und Aids [http://hivinsite.ucsf.edu/]

Expertensystem mit Informationen zu Resistenzen und Interaktionen bei HIV und Aids [www.radata.de] 